\title{
El modelo de producción y consumo en los últimos 20 años: Una revisión de la aproximación y las
} alternativas

\section{The production and consumption model in the last 20 years: A review of the approach and alternatives}

\author{
Fernando Corroto ${ }^{1}$ iD, Jesús Rascón Barrios ${ }^{2}$ (iD), Sara Martín-Hernanz $z^{3}$ [D
}

\section{RESUMEN}

En el presente trabajo se abordan cuestiones relacionadas con el modelo económico predominante en la actualidad y se reflexiona sobre sus efectos en los sistemas ambientales. En primer término, se realiza una descripción de los grandes componentes que lo sustentan, así como de su funcionamiento. A continuación se ofrece una visión crítica del modelo, detectando las imperfecciones e irracionalidades y resaltando la importancia del ciclo de producción y consumo como bucle de retroalimentación positiva promovido por la publicidad, el crédito y la caducidad. Todas las críticas se apoyan en la existencia de los límites medioambientales planetarios, razón definitiva para replantearse la viabilidad a medio y largo plazo del sistema económico. Para mostrar la gravedad en la que está sumido el planeta Tierra, se describe la huella ecológica como indicador cuantitativo del impacto que ejerce el hombre sobre el medio. Por último, se desarrolla la parte del trabajo centrada en la propuesta de decrecimiento como la alternativa más factible para mantener el equilibrio dinámico natural del globo terráqueo.

Palabras claves: Producción, consumo, obsolescencia, huella ecológica, Decrecimiento, cambio climático.

\begin{abstract}
The main issue treated in this paper is the economic model that dominates these days and its effects on the environment. Firstly, a thorough description of its great components is provided, as well as an explanation of the mechanisms that determine its behavior. After this first approach, we analyze the topic from a critic point of view in order to detect the main problems, paying special attention to the importance of the chains of producers and consumers as a positive feedback promoted by advertising, credit and caducity. Every statement is based in the environmental limitations, main reason to consider the half and long term viability of the economic system. This problematic situation is highlighted by the ecological footprint, as it is a quantitative indicator of the anthropological impact over the environment. To conclude the paper, we propose Decreasement, as the most suitable alternative to preserve the natural and dynamic equilibrium of the planet.
\end{abstract}

Keywords: Producers, consumers, obsolescence, ecological footprint, Decreasement, climate change.

\footnotetext{
${ }^{1}$ Universidad Autónomo de Madrid, Facultad de Ciencias, Edificio de Biología, Calle Darwin N ํ2, Ciudad Universitaria de Cantoblanco, Madrid, España.

${ }^{2}$ Universidad Nacional Toribio Rodríguez de Mendoza de Amazonas (UNTRM-A), Instituto de Investigación para el Desarrollo Sustentable de Ceja de Selva, Calle Higos Urco N³ 342-350-356, Calle Universitaria $N^{\circ} 304$, Chachapoyas, Perú

${ }_{3}^{3}$ Universidad de Sevilla, Departamento de Biología Vegetal y Ecología, Facultad de Farmacia, Calle Profesor García González N², Sevilla, España.

"Autor de correspondencia. Email: sara.martin.hernanz@gmail.com
} 


\section{INTRODUCCIÓN}

El crecimiento económico internacional, acelerado por la expansión demográfica humana y la evolución de las tecnologías, está en el centro de la crisis sin precedentes que atraviesa actualmente la biosfera, acompañada de una crisis económica y social (Asara et al., 2015). El sistema económico debe respetar los límites ecológicos globales ligados a la capacidad de carga de los ecosistemas, a la productividad primaria, a la integridad de la biodiversidad, a la estabilidad de los ciclos biogeoquímicos y al equilibrio del sistema climático del globo. En definitiva: tiene que respetar la salud, la estabilidad dinámica (Homeostasia) del complejo sistema geofisiológico de la biosfera, que James Lavelock y Lynn Margulis denominan Gaia (Asara et al., 2015). En la actualidad los criterios ecológicos comienzan a adquirir relevancia frente a una visión reduccionista en la que prevalecían los beneficios monetarios inmediatos (Sampedro, 2002). Para que las condiciones medioambientales se mantengan, debe plantearse un sistema basado en el decrecimiento económico de los países occidentales, pues si no se decrece voluntaria, racional, solidaria y ecológicamente, tendrá que hacerse llevado por las circunstancias de carestía de energía y cambio climático que acompañan al hundimiento del capitalismo global (Taibo, 2019).

\section{APROXIMACIÓN AL MODELO ECONÓMICOACTUAL}

\section{Mercado y Globalización}

El mercado en economía es entendido como cualquier conjunto de transacciones o acuerdos de negocios entre compradores y vendedores. En la teoría del mercado de competencia perfecta, cuyo creador fue el economista escocés Adam Smith en el siglo XVIII, se entiende que todos los participantes están plenamente informados de cuanto se ofrece o se demanda, a precios también conocidos, por lo que los ofertantes eligen el precio al que venden sus productos, de la misma manera que los demandantes eligen el producto al mejor precio. Además, la competencia entre los ven- dedores de un mismo producto hará que se vean forzados a venderlo lo más barato posible, lo cual beneficiará al comprador. También se llevará a cabo una producción racional por parte de los empresarios, pues solo se producirán aquellos bienes demandados por el mercado, a fin de usar las materias primas y el trabajo humano con la máxima eficacia. Adam Smith apoyaba el libre mercado haciendo alusión a una mano invisible que convierte la acumulación de egoísmos individuales en el máximo altruismo colectivo, por lo que cualquier autoridad en el libre mercado anularía la virtud de la mano invisible y resultaría siempre perjudicial (Sampedro, 2002). En el mercado real esta teoría se desmonta, en tanto que el comprador no puede elegir el precio ya que no tiene información sobre todos los vendedores presentes. Además, el comprador está influido por eficaces técnicas publicitarias, quedando su libertad condicionada, y pasando a demandar productos antes no usados que le han sido "revelados" por la publicidad o por la presión social. Los vendedores tampoco tienen información completa sobre la dispersa demanda ni sobre los planes de los compradores. Por otro lado, el ajuste automático entre la oferta y la demanda para lograr un precio óptimo no se da en el mercado real, debido a la fuerte desigualdad en la distribución de la riqueza dentro del sistema de mercado (Sampedro, 2002).

Una de las mayores imperfecciones del mercado real es la existencia de monopolios. Cuando una empresa vendedora logra acaparar una parte importante de la oferta, le permite imponer condiciones sobre el precio o las condiciones de contratación. Gracias a sus excepcionales medios técnicos y financieros dominantes consiguen, además, créditos y concesiones públicas o privadas privilegiadas. En Estados Unidos, por ejemplo, empresas como Sunkits, la cerveza Miller, las sopas Campbell's, McDonald's o MyM reciben todos los años del Market Acces Program unos 100 millones de dólares para la publicidad de sus productos en el extranjero. Los gobiernos otorgan subvenciones a la economía globalizadora de manera indirecta mediante inversiones en la infraestructura necesaria para una 
economía basada en el comercio. Por ejemplo es el caso subvenciones para la instalación de redes de transporte de larga distancia, las infraestructuras productoras de energía y las redes de comunicación de información ultrarrápida. La consecuencia de tal sistema de subvenciones directas e indirectas es que el precio de las mercancías transportadas alrededor del mundo es artificialmente barato, ya que ni la polución causada por el transporte ni el coste de las infraestructuras que hacen posible ese transporte se tienen en cuenta en el precio (Norberg-Hodge, 2006). La extraordinaria expansión planetaria de estas empresas refuerza al máximo la variedad y el alcance de sus decisiones. Gracias a ellas distribuyen sus operaciones según los criterios más convenientes, desplazando fábricas a países con salarios bajos, trasvasando fondos y contabilidades, eludiendo fiscalidades y legislaciones incómodas, aplastando a rivales locales y consiguiendo cifras de beneficios superiores a muchos presupuestos nacionales. Esta grave imperfección del mercado real basta para justificar intervenciones externas correctoras en defensa del interés público por motivos tanto económicos como éticos, sociales y medioambientales (Sampedro, 2002). El poder de las multinacionales se manifiesta en el hecho de que de las 100 mayores entidades económicas del mundo, 51 son empresas y 49 son países (Leonard, 2008). Además, el $70 \%$ del conjunto del comercio mundial está bajo el control de 500 empresas comerciales, y sólo dos de ellas, Cargill y Archer Daniels Midland, controlan entre el $70 \%$ y el $80 \%$ del comercio de cereales. General Motors y Ford tienen, sólo entre ambas, ventas anuales muy superiores al PIB de la totalidad del África subsahariana (Norberg-Hodge, 2006). Lo lógico sería que los gobiernos reglamenten y graven las importaciones, favoreciendo así la industria del país y protegiendo también la estabilidad económica, el nivel de vida de los ciudadanos y su medio ambiente (Norberg-Hodge, 2006).

La economía del sistema internacional moderno se vincula a dos condicionantes: la posibilidad prácticamente instantánea de comunicaciones y de transferen- cias económicas, y una amplia liberación de las operaciones privadas y ausencia de control sobre ellas. Ésta es, en síntesis, la estructura a la que ha llegado el mercado en su evolución reciente, a la que se ha dado el nombre de Globalización. La globalización es una realidad; para unos positiva, pues será el fenómeno que rompa las desigualdades entre ricos y pobres, e inevitable, pues es causa directa del imparable avance tecnológico; pero negativa para otros, pues las diferencias económicas cada vez son más marcadas entre el Norte y el Sur, y la globalización que aproximaría el planeta a uno más equitativo debería ser más genérico, con unos componentes social, cultural, económico y tecnológico igualitarios (Sampedro, 2002).

La Globalización ha facilitado el crecimiento de las grandes multinacionales, y éstas a su vez han ganado terreno a los gobiernos, y han desarrollado eficaces estrategias para producir bienes a bajos precios y promover un consumo desmesurado, las cuales permiten el mantenimiento del ciclo de producción y consumo que se explica a continuación.

\section{Economía de los materiales}

El corolario del crecimiento económico es el crecimiento "material", que está completamente ligado a los impactos ecológicos (Schneider, 2006). La economía de los materiales hace alusión al sistema por el que los bienes sufren un ciclo de extracción, producción, distribución, consumo y eliminación. Este sistema lineal está en crisis, debido a la existencia en el planeta con límites medioambientales. El funcionamiento de la economía de materiales es posible gracias a los trabajadores, los gobiernos y las empresas (Leonard, 2008).

Este ciclo se sustenta por tres elementos: la publicidad (a partir de la cual nace en los consumidores el deseo de poseer bienes y servicios prescindibles), el crédito (que permite, aun en ausencia de recursos, que podamos comprar esos bienes o servicios que no necesitamos), y, por último, la caducidad (los bienes son producidos de tal manera que en un periodo de tiempo muy breve dejan de funcionar). Este último elemento es lo que algunos autores llaman obsolescencia (Cue- 
va y Ruesta, 2019).

\section{Extracción}

La extracción o explotación de recursos naturales consiste en la obtención de materias primas con las que, a partir de la producción, se obtendrán los bienes materiales. La extracción es la causa principal del agotamiento de los recursos naturales, y por tanto uno de los mayores problemas ambientales que propician el Cambio Global. La extracción de recursos naturales se lleva a cabo en las sociedades occidentales sin considerar el tiempo necesario para su renovación. Para expresar la gravedad del asunto, basta con decir que en EEUU quedan menos del $4 \%$ de bosques primarios y el $40 \%$ de los cursos de agua ya no son aptos para beber. Hay un 5\% de la población mundial en EEUU, y sin embargo usan el 30\% de los recursos del mundo y generan el 30\% de los desechos (Leonard, 2010). Además, la mayoría de recursos que EEUU extrae son del Tercer Mundo. Otros datos alarmantes son que el 75\% de los recursos pesqueros del mundo actualmente está siendo explotado al límite de su capacidad o sobreexplotados, el $80 \%$ de los bosques primarios del mundo ha desaparecido, tan sólo en la Amazonía estamos perdiendo 2000 árboles cada minuto (Leonard, 2008).

\section{Producción}

Consiste en la transformación de las materias primas obtenidas de recursos naturales a través del uso de energía y de determinados compuestos químicos tóxicos. Hoy en día hay más de 100.000 químicos sintéticos en uso en el mercado, de los que sólo un pequeño porcentaje ha sido investigado para al menos un potencial efecto en la salud, como cáncer, toxicidad reproductiva, toxicidad para el desarrollo, o impactos en el sistema inmune. Incluso cuando se investiga, cada químico se prueba de manera independiente en vez de en forma combinada. Estos tóxicos se acumulan en la cadena alimentaria y se concentran en nuestro cuerpo. De hecho, desde que nacemos estamos expuestos a estos tóxicos mediante el consumo de leche materna, alimento que presenta los niveles más altos de varios contaminantes tóxicos. Los afectados por estos compuestos tóxicos no solo lo son los consumidores, si no, y en mayor medida, los trabajadores de la producción (Leonard, 2008).

En Estados Unidos, la industria reconoce que emite alrededor de 2 mil millones de kilogramos de químicos tóxicos al año. Por esta razón se trasladan fábricas contaminantes a otros países, aunque mucha de esa contaminación se extiende por toda la superficie del planeta movida por las corrientes atmosféricas (Leonard, 2008).

\section{Distribución}

Es la fase del ciclo en la que los bienes y servicios producidos por la empresa lleguen a los consumidores. Las empresas tratan de externalizar los costes, es decir, que el coste real de producir los bienes no se vea reflejado en los precios. Para ello no se paga la extracción del recurso, los trabajadores de la extracción cobran bajos salarios, no se valora la pérdida de salud de los mismos, no se palian los problemas asociados a la contaminación que supone el traslado de materias primas o de bienes ya producidos, etc., todo con el fin de que los consumidores obtengan bienes a precios absurdos, que permitan un constante consumo, y un mantenimiento del ciclo de la economía de materiales (Leonard, 2008).

El transporte, ya sea de materias primas destinadas a la producción, o de los bienes dispuestos para el consumo, suponen múltiples impactos ambientales en todas las escalas geográficas, desde el cambio climático (global), hasta la lluvia ácida (regional), el ruido y el smog (local). Los impactos ambientales están estrechamente ligados a los modos de transporte, al enorme consumo de combustibles fósiles asociado a las emisiones generadas y a la misma infraestructura del transporte. El 40\% del comercio mundial se realiza a través de tráfico aéreo, por lo que es el que mayor consumo de energía conlleva (Garrido, 1999).

\section{Consumo}

El consumo es la obtención de un bien o servicio a cambio de pagar un precio monetario por el mismo. Dos de las estrategias más efectivas para que se promueva el consumo son: la obsolescencia programada y la obsolescencia percibida. 
La obsolescencia programada consiste en planificar el fin de la vida útil de los productos. Esta estrategia la consiguen las empresas utilizando materiales más frágiles o de menor calidad, poniendo piezas defectuosas o incluso implantando chips para que el aparato electrónico deje de funcionar en un determinado momento. Algunos autores han denominado a la obsolescencia programada "el motor secreto de nuestra sociedad de consumo". De hecho, la obsolescencia programada surgió al mismo tiempo que la producción en masa y que la sociedad de consumo, poco después de la Revolución Industrial (Slade, 2007). Un caso ilustrativo de obsolescencia programada se remonta a 1924; cuando las empresas fabricantes de bombillas limitaron la vida útil de las bombillas a 1000 horas (Metze, 2004).

La obsolescencia programada deja graves secuelas para el medioambiente ya que no se tiene en cuenta el gasto innecesario de recursos utilizados. Pero el principal problema que crea la obsolescencia programada es que provoca un flujo constante de residuos, los cuales, en muchos casos, acaban en el Tercer Mundo. Uno de los países que más ha sufrido este problema ha sido Ghana. Este país recibe cada año toneladas de residuos electrónicos, de productos que se han quedado obsoletos o no funcionan. Estos residuos son arrojados en vertederos. Por ejemplo, el vertedero de Agbogbloshie antes de ser un vertedero era un río con gran cantidad de peces donde los niños jugaban en sus orillas. Hoy en día, es el vertedero más grande del país y allí van los jóvenes de familias paupérrimas en buscar de chatarra. Los países desarrollados hacen estos envíos bajo el lema de "queremos cerrar la brecha digital entre Europa, América y el resto de África", pero lo cierto es que más del $80 \%$ de estos productos no funcionan ni pueden ser reparados. Además de un problema medioambiental es un problema social ya que la gente de estos países en vías de desarrollo tienen que ver y sufrir como su país se convierte en el vertedero del planeta, donde echar los desperdicios de los países desarrollados (Latouche, 2007).

La obsolescencia percibida es la que convence a los consumidores para desechar bienes que todavía son perfectamente útiles. Esto se consigue cambiando el aspecto de los objetos, de tal manera que en poco tiempo se queden anticuados. La publicidad, y los medios de comunicación, desempeñan un importante papel en la obsolescencia percibida, mostrando a los consumidores sus defectos y como pueden resolverlos mediante el consumo (Leonard, 2008).

Otro fenómeno que promueve el consumo es el efecto rebote, el cual se define como un aumento del consumo de un producto o servicio debido por la reducción de su precio de coste. El efecto rebote es algo intencionado para incrementar las ventas y los beneficios mediante el aumento de la demanda (Schneider, 2006).

\section{Eliminación de residuos}

E1 99\% de los bienes que circulan a través de este sistema es basura en menos de 6 meses. En EEUU se generan dos kilogramos de basura por hogar al día, el doble de lo que se producía hace treinta años. La basura, ya sea enterrada en un vertedero, o primero incinerada y luego enterrada, contamina el aire, el suelo, el agua y contribuye al cambio climático. La incineración libera todos los compuestos tóxicos utilizados en la producción a la atmósfera y genera nuevos supertóxicos, como las dioxinas. Muchas empresas exportan, además de la producción, los vertederos y las incineradoras, a países del Sur (Leonard, 2008).

Una de las soluciones propuestas para disminuir la cantidad de desechos generados es el reciclaje. No obstante, reciclar no es suficiente porque de cada cubo de basura que se genera en el hogar, se generaron 70 cubos de basura en la producción de los bienes materiales (Leonard, 2008).

\section{CRÍTICAS AL MODELO ECONÓMICO ACTUAL DESDE UNA PERSPECTIVA ECOLÓGICA}

El sistema de producción y consumo imperante en la actualidad promueve el crecimiento económico, pero a costa de los recursos naturales presentes en el planeta Tierra, que tiene unos límites medioambientales cada vez más acusados, puesto que no se considera el tiem- 
po necesario para la renovación de los recursos. El consumo de productos y servicios requiere recursos nuevos (energía, materias primas, espacio) que están en la base del cambio climático, la polución, el descenso de la biodiversidad, la destrucción de los espacios naturales, la degradación medioambiental, los peligros nucleares e incluso los genéticos. El problema no es tanto la penuria de recursos como los estragos provocados por la utilización del espacio, la puesta en circulación en el sistema económico y las emisiones (Schneider, 2006).

Más de las tres cuartas partes de los recursos energéticos que utilizamos hoy en día son de origen fósil: el gas, el petróleo, el uranio y el carbón son recursos no renovables, o más exactamente recursos con unas tasas de renovación extremadamente bajas. Al ritmo de consumo actual, restan 41 años de reservas petrolíferas, 70 años de gas y 25 años de uranio. El consumo de recursos supera al descubrimiento de otros nuevos. Además se prevé que en el plazo de 20 años se dupliquen en el mundo tanto el parque automovilístico como el consumo energético. Aun así, parece que el mayor peligro al que debemos enfrentarnos hoy es el de los daños que infligimos al clima, más acechante que el agotamiento de los recursos naturales (Cleméntin y Cheynet, 2006).

Además de la contradicción de que el modelo actual se base en producción y consumo ilimitado en un planeta finito, el sistema económico presenta otras incoherencias e imperfecciones: El modelo económico actual es justificado con argumentos como el aumento de la cohesión social, la igualdad, el empleo y el asentamiento de los servicios públicos cuando se produce crecimiento económico. Bastan los motivos para afirmar que no son más que supersticiones: China ha crecido espectacularmente en las dos últimas décadas sin que se encuentre hoy socialmente más cohesionada que en el pasado, el crecimiento operado en los países ricos se ha realizado en muy buena medida a costa del expolio de los recursos humanos y materiales de los Estados más pobres, el crecimiento español en los últimos decenios ha generado un aumento significati- vo en la cifra de desempleados, los servicios públicos se están privatizando de una manera cada vez más evidente, y, lo que es más preocupante, el crecimiento se traduce en un continuo de agresiones medioambientales irreversibles y en un agotamiento de los recursos naturales (Taibo, 2019).

Ante esta situación de Crisis Global, los Gobiernos y los organismos internacionales han tomado medidas insuficientes, como las inmersas en el protocolo de Kyoto y en acuerdos afines, que no tratan el problema de raíz, y que, en el mejor de los casos, retrasan levemente en el tiempo la aparición de los problemas reales (crisis social, económica y medioambiental) (Eschenhagen y Maldonado, 2014).

Otra imperfección del sistema económico es utilizar el producto interior bruto (PIB) como índice de desarro1lo, pues éste no es más que el valor de los productos y servicios intercambiados (Schneider, 2006). La destrucción de recursos naturales contribuye al PIB, mientras que su preservación, a pesar de garantizar en paralelo sutiles equilibrios medioambientales y derechos de las generaciones venideras, no se contabiliza y por tanto no contribuye al PIB (Taibo, 2019). Un ejemplo de esto es el hecho de que la destrucción de la selva amazónica es una buena noticia en términos de PIB, mientras que no su conservación. Del mismo modo, cuando contaminamos, estamos permitiendo un doble crecimiento del PIB, por ejemplo los atascos de tráfico al propiciar un mayor consumo de gasolina (Cueva y Ruesta, 2019). Podría decirse que el consumo de los recursos sigue de forma casi lineal al PIB (Schneider, 2006).

A partir de datos estadísticos registrados en algunos países se ha constatado que la felicidad no tiene que ver con el crecimiento económico. Tal es el caso de Estados Unidos, país en el cual la renta per cápita hoy registrada es tres veces mayor que la contabilizada al final de la Segunda Guerra Mundial. Sin embargo, en 2005 un 49\% de los encuestados declaraba ser cada vez menos feliz, frente a sólo un $26 \%$ que afirmaba lo contrario (Iranzo, 2015). 
IV. UN INDICADOR DE LOS EFECTOS DEL SISTEMA ECONÓMICO SOBRE LOS SISTEMAS NATURALES: LA HUELLA ECOLÓGICA

\section{Concepto}

La huella ecológica es una importante herramienta para definir tanto el impacto de las actividades humanas sobre el ecosistema, como las medidas correctoras para paliar dichos impactos (Doménech, 2010). El concepto de huella ecológica fue definido por Wackernagel y Rees (1996) como: "el área de territorio ecológicamente productivo (cultivos, bosques o ecosistemas acuáticos) necesaria para producir los recursos utilizados y para asimilar los residuos generados por una población determinada con un nivel de vida específico de forma indefinida y en cualquier área". Por tanto, podemos decir que la huella ecológica es un indicador global ya que transforma cualquier tipo de consumo (toneladas, kilowatios, litros, etc.) y de residuo producido, en un único número íntegramente significativo.

Generalmente, el sistema de cálculo de la huella ecológica se basa en determinar la cantidad de terreno necesario para producir los bienes consumidos y para absorber los residuos producidos; una vez calculada tal superficie la huella ecológica se obtiene dividiendo el terreno productivo total por el número de habitantes en hectáreas per cápita (Miguélez Pose, 2003). Para calcular estas superficies, la metodología se basa en dos aspectos básicos: por un lado la contabilización del consumo de los diferentes elementos en unidades físicas, y por otro la transformación de éstos consumos en superficie biológica productiva apropiada a través de índices de productividad.

Los tipos de terreno productivo que se consideran para este cálculo serían cultivos, pastos, bosques, mar, terreno construido, área de absorción de $\mathrm{CO} 2$ y reserva para biodiversidad (según la metodología original de Wackernagel y Rees (1996) considera la propuesta de reservar un $12 \%$ de la superficie del territorio para la preservación de esta biodiversidad). La suma de todos ellos es la capacidad de carga local y está expresada en hectáreas por habitante.

\section{Déficit ecológico}

El concepto de huella ecológica viene íntimamente ligado y contrapuesto al de capacidad de carga (Martín-Palmero et al., 2004); la capacidad de carga se define como la capacidad que tiene un ecosistema para sustentar y mantener al mismo tiempo la productividad, adaptabilidad y renovabilidad de recursos; por lo tanto podemos decir que la comparación entre la huella ecológica de un país o región y su capacidad de carga nos dirá si dicha economía es autosuficiente y/o presenta un déficit ecológico.

\section{Huella ecológica de las naciones mundiales}

Si observamos los datos de huella ecológica a nivel mundial de diferentes zonas geográficos (Figura 2) vemos que la diferencia que existe entre el hemisferio Norte y el Sur es muy acusada (los datos son aproximados)

En la figura 2 puede observarse que las regiones de mayor riqueza (América del Norte y Europa Occidental) son las que mayor apropiación del terreno presentan. Cada estadounidense y casa canadiense hacen uso de 9,4 hectáreas, más de cinco veces la media mundial que podría permitirse para la viabilidad del planeta. En la Unión Europea la cifra correspondiente es de 4,8 hectáreas, en el resto de Europa de 3,7, en el Oriente Próximo de 2,2 y en América Latina de 2,0. Estos datos reflejan que una tercera parte de la población mundial es responsable del 60 por ciento de la huella ecológica (Taibo, 2019). Podría constatarse que las zonas de menor renta presentan una huella ecológica menor que aquellas en las que el PIB es más alto, las cuáles se apropian proporcionalmente de una mayor cantidad de terreno per cápita (Martín-Palmero et al., 2004).

En el Figura 3 se ilustra el porcentaje de cada uno de los recursos naturales utilizados en la producción de materiales medidos en términos de superficie que corresponden a la huella ecológica media mundial. Se comprueba que la principal demanda se debe a la absorción de $\mathrm{CO} 2$, con casi la mitad del terreno requerido (48\%). Siguiendo el gráfico se aprecia que el 5\% del terreno apropiado corresponde al mar, al igual que 
el terreno edificado. Los bosques ocuparían el 9\% y los cultivos y pastos el $29 \%$. Por último el consumo de energía nuclear e hidroeléctrica supone un $4 \%$ de la demanda. Estos porcentajes aportan una relevante información de cara a la propuesta de decrecimiento que se describirá a continuación.

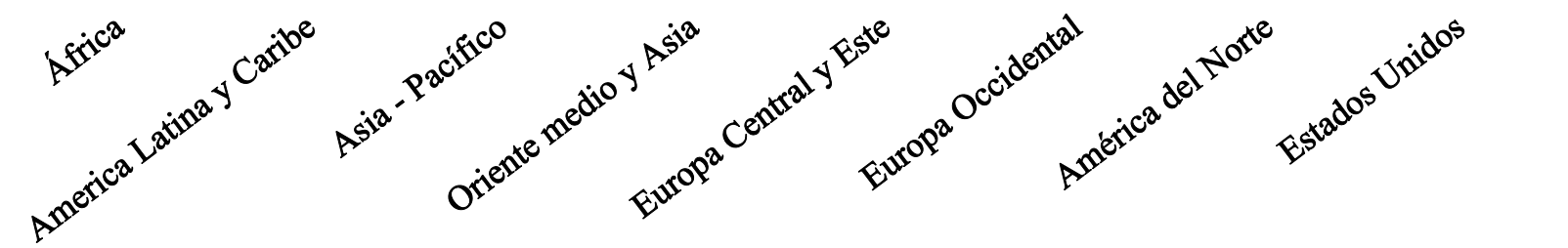

Figura 2. Valores aproximados de la huella ecológica de distintas regiones geográficas del mundo. Fuente: Venetoulis et al. (2004) modificado.

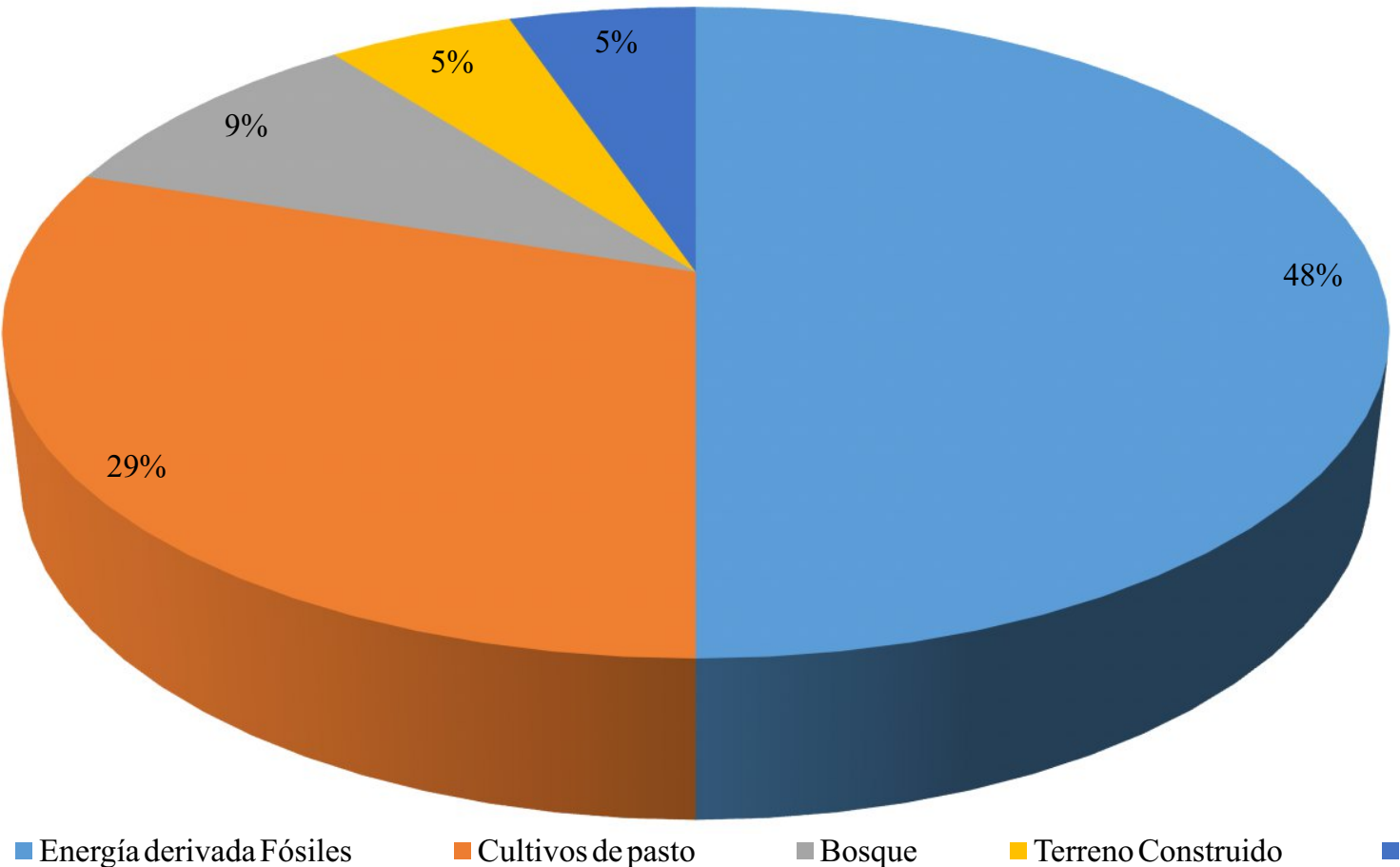

Energía derivada Fósiles

Bosque

Terreno Construido

- Mar 
En vistas al futuro, se prevé que en 2050 la población aumentará en el Tercer Mundo en un 60\%. Los países que lo conforman multiplicarían su consumo (actualmente relativamente débil) por 24 . El resultado es que hacen falta 12 planetas Tierra para satisfacer las necesidades de todos a largo plazo. De este modo, para reducir a la mitad el consumo mundial de recursos, habría que recudir 12 veces el consumo en los países ricos, mientras que los países del Tercer Mundo solo podrían doblarlo (Schneider, 2006).

\section{BÚSQUEDA DE ALTERNATIVAS: LA PROPUESTA DE EL DECRECIMIENTO}

Según la Declaración Universal de los Derechos Humanos "todos los seres humanos nacen libres e iguales en dignidad y derechos". Sin embargo, los países ricos representan un $20 \%$ de la población del planeta y consumen el $80 \%$ de los recursos naturales, es decir, aproximadamente 16 veces más por habitante que los países del Sur, por lo que a los países occidentales no les queda más remedio que reducir su producción y su consumo a fin de "decrecer" (Clémentin y Cheynet, 2006). Este modelo alternativo de decrecimiento exige un aumento de responsabilidad tanto política como del consumidor, y debe estar articulado en torno a tres ejes. El primero sería una economía de mercado controlada, para evitar cualquier fenómeno de concentración, lo cual representaría, por ejemplo, el fin del sistema de franquicia. Esta economía de pequeñas entidades, además de su carácter humanista, tendría el inmenso mérito de no generar publicidad. El segundo eje, la producción de equipos que precisen de una inversión, sería financiado por capitales mixtos privados y públicos, controlados por los políticos. El tercer eje atañe a los servicios públicos esenciales, que tendrían como característica el no ser privatizables (acceso al agua, a la energía disponible, a la educación y a la cultura, a los transportes públicos, a la sanidad y a la seguridad de las personas). La puesta en práctica de un modelo así supondría la expansión de un comercio real equitativo, es decir, unas condiciones de remuneración y protección social idénticas en los países consumidores y en los productores, y conllevaría el fin de la esclavitud y del neocolonialismo (Clémentin y Cheynet, 2006).

La alternativa del decrecimiento, además de reclamar las reducciones significativas en los niveles de producción y de consumo en los países del Norte opulento, exige la reorganización de las sociedades occidentales sobre la base de principios y valores muy diferentes de los actuales. Estos principios y valores son, en primer caso, la primacía de la vida social frente a las lógicas de la producción, de la propiedad, del consumo y de la competitividad; en segundo lugar, el ocio creativo frente a las formas habituales de ocio, siempre vinculadas con el consumo; el siguiente hace referencia al reparto del trabajo, el cuarto exige el establecimiento de una renta básica a todos los habitantes del planeta, el quinto reclama la necesidad de reducir las dimensiones de muchas infraestructuras productivas, administrativas y de transporte; el sexto postula la recuperación de lo local, la relocalización, frente a lo global; el sétimo y último consiste en asumir la simplicidad voluntaria, que implica reducir la dependencia con respecto al dinero, el consumo y la velocidad, hacer un uso sensiblemente mayor de los medios colectivos y comunitarios y acrecentar la equidad en todos los órdenes (Taibo, 2019).

Lo más sensato, llegados a este escenario, sería promover una economía saludable, que se define como un modelo económico que, como poco, no recurra al capital natural. La idea de este modelo es restituir el capital natural ya destruido, puesto que vivir de las rentas de la naturaleza ya constituye un desafío extraordinario. El padre del decrecimiento, Nicholas Georgescu-Roegen, opina que en cada ocasión que recurrimos a nuestro capital natural estamos hipotecando las posibilidades de supervivencia de nuestros descendientes (Clémentin y Cheynet, 2006).

Edward Goldsmith, el fundador de la revista The ecologist, aventura que con un mínimo de voluntad política, reduciendo en un $4 \%$ durante 30 años la producción y el consumo, habría alguna posibilidad de escapar de la crisis climática. Pero es importante que el decreci- 
miento sea sostenible, es decir, que no genere una crisis social que cuestione la democracia y el humanismo. Si se aplicara el decrecimiento, el primer objetivo sería la transición de la energía fósil, quedando reservada a usos de supervivencia, por ejemplo en el campo de la medicina, lo cual conllevaría la relocalización de los mercados, tanto en su producción como en su consumo, pues la mano del obra barata del Tercer Mundo ya no resultaría accesible, por lo que aumentaría el número de empleados en los países occidentales. El problema no se plantea para la mayoría de los habitantes del globo, pues el $80 \%$ de los humanos vive sin automóvil, sin nevera y sin televisión; y el 94\% de los humanos no ha tomado nunca un avión (Clémentin y Cheynet, 2006). Existe, a su vez, una necesidad de acabar con la actividad de sectores como el de la industria militar, el automovilístico, el de aviación, el de la construcción o el de la publicidad, lo cual se traduciría inevitablemente en una reducción del producto interior bruto (Taibo, 2019).

Latouche propone diez medidas mínimas que deberían aplicarse en los países del Norte con el fin de decrecer:

1. Rebajar la huella ecológica en los países del Norte para permitir que ésta crezca en los países del Sur.

2. Reducir las operaciones de transporte - son responsables de un 30 por ciento de las emisiones de $\mathrm{CO}_{2}-\mathrm{y}$ aplicar ecotasas.

3. Relocalizar las actividades.

4. Restaurar la agricultura tradicional, acabando, por ejemplo, con los pesticidas.

5. Reducir el tiempo de trabajo y crear empleos.

6. Relanzar la 'producción' de bienes relacionados la sabiduría, la solidaridad, el amor...- no mercantiles, que pueden consumirse sin perjudicar a los demás.

7 . Reducir el gasto de energía.

8. Restringir notablemente el espacio publicitario.

9. Reorientar la investigación tecno-científica.

10. Reapropiarse del dinero y -a través, por ejemplo, de mutualidades locales y municipales- no dejarlo en manos de los bancos

\section{CONCLUSIONES}

La Crisis Global que está sufriendo desde mediados del siglo XX el planeta Tierra es cada vez más evidente. La actual crisis económica y social no debe distraer la atención de la brutal crisis medioambiental, ya que la combinación de los diversos impactos generados por el ser humano y promovidos por la economía de los materiales ha llegado a un punto de no retorno, y solo un cambio de mentalidad ciudadana de los países occidentales puede poner freno a la destrucción de la especie humana y del planeta. La existencia de límites medioambientales es razón indudable para que se fomente el respeto por dichos límites por parte de todos los habitantes, y no cabe duda de que en el modelo económico actual esta necesidad no se está considerando, de tal manera que se está sobrepasando con creces la capacidad de carga de los ecosistemas.

Las estrategias de las multinacionales permiten producir bienes a bajo coste y un creciente consumo de productos por parte de la población occidental. Esto es posible a costa de la explotación de los trabajadores y de la generación de impactos sobre el medio ambiente en todas las fases del ciclo: la fase de extracción provoca el agotamiento de recursos naturales sin tener en cuenta su tasa de renovación, la fase de producción y distribución genera gases contaminantes que contribuyen al cambio climático como resultado del uso de combustibles fósiles, y el desproporcionado consumo genera gran cantidad de residuos, junto con los procedentes de la fase de producción. Una de las bases del consumo actual es la obsolescencia programada, y contribuye fuertemente a la acumulación de residuos. Un problema añadido del mantenimiento del sistema de producción, es el uso de una alta proporción de recursos energéticos de origen fósil, que tienen baja tasa de renovación y su combustión genera la emisión de gases contaminantes que propician el cambio climático. Huelga decir que son múltiples los efectos negativos derivados del modelo económico actual. No obstante, los gobiernos están empeñados en aumentar su PIB año tras año, estando el PIB muy relacionado con el consumo de recursos, pero nunca con su conser- 
vación.

La manera más ilustrativa de demostrar el impacto sobre el medio a nivel individual es la huella ecológica, que refleja por un lado, como desde mediados del siglo XX hemos sobrepasado la capacidad de carga del planeta, y por otro lado las enormes diferencias en términos de huella ecológica que se producen en el planeta, siendo una tercera parte de la población mundial responsable del $60 \%$ de la huella ecológica, esta tercera parte correspondiendo, como es de esperar, a los países con mejores valores en términos de PIB.

Conocidas y descritas algunas de las muchas situaciones que se están produciendo en la actualidad como consecuencia del crecimiento económico, la globalización y las estrategias mercantiles, todas ellas íntimamente relacionadas, pensamos que no queda más remedio que replantearse el modelo, utilizar el sentido común y que se produzca un cambio de mentalidad global que permita instalar valores éticos y de respeto hacia el medio ambiente, siendo éste último el que condiciona nuestra permanencia a medio y largo plazo en el planeta. Para ello creemos fielmente en la propuesta de El Decrecimiento como única vía de convivencia real la naturaleza y de redistribución equitativa de los recursos en todo el mundo. Creemos que no es imposible ni está evocado al fracaso obligar a los gobiernos a cambiar la situación, puesto que simplemente exige que un número suficiente de personas tome conciencia del desastre de la situación actual. Es preciso que las políticas gubernamentales den prioridad a la pequeña escala frente a la gran escala, para permitir así la expansión y el desarrollo de más economías enmarcadas en lo local y la destrucción de las multinacionales que tantos daños están generando en todas las dimensiones planetarias. Pero la solución no está solo en manos de los gobiernos, si no en la sencillez voluntaria que nos permita escapar de la esclavitud del consumo.

\section{REFERENCIAS BIBLIOGRÁFICAS}

Asara, V., I. Otero, F. Demaria, y E. Corbera. 2015.

"Socially sustainable degrowth as a social-ecological transformation: repoliticizing sustainability." Sustainability Science 10 (3): 375-384.

Clémentin, B., y V. Cheynet. 2006. “Introducción: El decrecimiento sostenible." En Objetivo Decrecimiento. ¿Podemos seguir creciendo hasta el infinito en un planeta finito?. Cheynet, V., B. Clémentin, S. Latouche, M. Bonaiiuti, F. Schneider, J. Grinevald, H. Norberg-Hodge, W. Hoogendik y D. Cheynet (eds). Barcelona (España): Leqtor.com

Cueva, W. C. E. y I. M. Ruesta. 2019. "Necesidad de regulación legal de la obsolescencia programada en el Perú." EPISTEMIA 1 (1): 11-18.

Doménech, J. L. 2010. La huella ecológica y desarrollo sostenible. Madrid (España): Ediciones AENOR.

Eschenhagen, M. L., y C. E. Maldonado. 2014. Un viaje por las alternativas al desarrollo: perspectivas y propuestas teóricas. Bogotá (Colombia): Editorial Universidad del Rosario.

Garrido, J. 1999. "Impactos medioambientales y sociales del transporte." Geographicalia (37): $37-51$.

Iranzo, J. M. 2015. “Crisis económica, decrecimiento y rituales de interacción: Un camino a la sostenibilidad." Papeles del CEIC. International Journal on Collective Identity Research (1): $1-31$.

Latouche, S. 2007. La otra África: autogestión y apaño frente al mercado global. Barcelona (España): Ediciones Asociación Cultural Oozebap

Leonard, A. 2008. La historia de las cosas. Madrid (España): Fondo de cultura económica de España.

Martín-Palmero, F., F. González-Laxe, F. MiguélezPose, E. Menéndez Pérez, y J Dopico-Castro. 2004. Desarrollo sostenible y huella ecológica. Una aplicación a la economía gallega. A Coruña (España): Netbiblo.

Metze, M. 2004. Anton Philips (1874-1950), They will 
know who they're dealing with. Amsterdam

(Holanda): Balans Publishers

Norberg-Hodge, 2006. "De la dependencia local a la interdependencia local." En Objetivo Decrecimiento. ¿Podemos seguir creciendo hasta el infinito en un planeta finito?. Cheynet, V., B. Clémentin, S. Latouche, M. Bonaiiuti, F. Schneider, J. Grinevald, H. Norberg-Hodge, W. Hoogendik y D. Cheynet (eds). Barcelona (España): Leqtor.com

Sampedro, J. L. 2002. El mercado y la globalización. Madrid(España): Editorial Destino.

Schneider, 2006. "Sin sobriedad no hay eficacia." En Objetivo Decrecimiento. ¿Podemos seguir creciendo hasta el infinito en un planeta finito?. Cheynet, V., B. Clémentin, S. Latouche, M. Bonaiiuti, F. Schneider, J. Grinevald, H. Norberg-Hodge, W. Hoogendik y D. Cheynet (eds). Barcelona (España): Leqtor.com

Slade, G. 2007. Made to Break: Technology and Obsolescence in America. Londres (Inglaterra): Harvard University Press

Taibo, C. 2019. El decrecimiento explicado con sencillez. Madrid (España): Editorial Los Libros de la Catarata.

Venetoulis, J., D. Chazan, y C. Gaudet. 2004. Ecological footprint of nations 2004. Oakland (EEUU): Redefining Progress.

Wackernagel, M. y W. Rees (1996). Our ecological footprint: reducing human impact on the earth. Gabriola (Canada): New Society Publishers. 